\title{
Simulated enhancement of solar type II radio bursts during the collision of two shocks associated with coronal mass ejections
}

\author{
J. I. Sakai ${ }^{1}$, T. Mori ${ }^{1}$, S. Saito ${ }^{1}$, Y. Tanaka ${ }^{1}$, and H. Aurass ${ }^{2}$ \\ 1 Laboratory for Plasma Astrophysics, Faculty of Engineering, University of Toyama, 3190, Gofuku, Toyama 930-8555, Japan \\ e-mail: sakaijun@eng.u-toyama.ac.jp \\ 2 Astrophysical Institute Potsdam, An der Sternwarte 16a, 14482 Potsdam, Germany
}

Received 2 March 2006 / Accepted 3 April 2006

\begin{abstract}
Aims. We investigate how solar type II radio bursts can be enhanced when two fast magnetosonic shocks associated with coronal mass ejections (CMEs) collide. This work was motivated by recent observations showing that the radio signature is in the form of intense continuum-like radio emission following an interplanetary type II burst, when a fast CME overtakes a slow CME.

Methods. We used a 2D3V fully relativistic, electromagnetic particle-in-cell (PIC) simulation.

Results. We found that during the collision between a fast shock with Alfvén Mach $M_{\mathrm{A}}=3$ and a slow wave with Alfvén Mach $M_{\mathrm{A}}=0.75$, the radio emission with a broad band can be enhanced with an amplitude about ten times larger than for the undisturbed type II burst. We also found that when a fast shock with Alfvén Mach $M_{\mathrm{A}}=3$ overtakes a slow shock with Alfvén Mach $M_{\mathrm{A}}=1.5$, the enhancement of the radio emission is about five times greater than the undisturbed type II burst.
\end{abstract}

Key words. plasmas - radiation mechanisms: non-thermal - shock waves - methods: numerical - Sun: radio radiation

\section{Introduction}

Radio observations of coronal and interplanetary shock waves (so-called solar type II radio bursts) are a unique tool testing models of particle acceleration at shock waves. In radio spectrograms, they frequently occur as slowly drifting lanes of fundamental and second-harmonic plasma emission (Smith 1970; Melrose 1980; Mann et al. 1995). In limb events, a third harmonic lane has also been reported (Zlotnik et al. 1998). The emission signature in a lane consists of a diffuse narrowband feature (the backbone, frequently showing band split). Vršnak et al. (2001, 2002) analysed the band split of coronal (high frequency) and interplanetary (low frequency) type II bursts and found consistent behavior in both frequency ranges. In coronal type II bursts the backbone is frequently superposed with bundles of negatively and positively drifting type III-like (beamdriven) bursts starting in the backbone, which are called herring bones (Mann \& Klassen 2005). To our knowledge this fine structure effect has not yet been systematically analysed using low frequency radio spectra, but we have no doubt that it exists there, too. Bale et al. (1999) reports the first in-situ observation of the source region of an interplanetary type II burst and found velocity-dispersed electron beams there and spatial structure possibly related to the band-split effect.

A general picture of emission from type II shocks suggests that the emission mechanism is plasma emission near the fundamental and second harmonics (Smith 1970; Melrose 1980). All the characteristic nonthermal type II burst radio signatures together remotely evidence electron acceleration at coronal and interplanetary shock waves. Shock-excited Langmuir waves may be converted into escaping radio waves by nonlinear wave-wave processes (see reviews by Goldman \& Smith 1986;
Cairns et al. 2003; Aschwanden 2004). Recent observations from the Ulysses spacecraft (Simnett 2003) have measured the energy spectrum of the ambient ions associated with the passage of a fast magnetosonic quasi-perpendicular shock driven by a coronal mass ejection (CME). Near the shock front the energy spectrum of the accelerated ions, which are predominantly protons, exhibit features (maxima) at about $250 \mathrm{KeV}-1.5 \mathrm{MeV}$. Simnett et al. (2004) show that the protons with energy of about $1 \mathrm{MeV}$, which corresponds to $\left(m_{\mathrm{i}} / m_{\mathrm{e}}\right) V_{\mathrm{A}}\left(M_{\mathrm{A}}-1\right)^{3 / 2}$, can be accelerated by the surfatron mechanism (Sagdeev 1996) of a fast magnetosonic shock wave propagating almost perpendicular to an ambient magnetic field. The in situ measurements therefore directly illustrate that this mechanism is very probably responsible for interplanetary particle acceleration.

Recently, Sakai et al. (2005) addressed the formation of shock waves driven by CME, the acceleration process of both protons and electrons and the emission mechanism of electromagnetic waves (solar type II bursts) from the shock front, by using a two-dimensional, fully relativistic particle-in-cell (PIC) simulation. They showed that, for fast magnetosonic shock waves with $M_{\mathrm{A}} \geq 2.0$ and a propagation angle between 90 and 70 degrees, protons near the shock front can be accelerated by the surfatron acceleration mechanism, while the emission of the electromagnetic waves from the shock front was observed almost independently of the Alfvén Mach and the propagation angles. For perpendicular propagation, they also found that the electron Bernstein waves are generated near the shock front and can be converted to the electromagnetic waves (type II radio bursts) through direct linear mode conversion. It was also found from the oblique propagation that near the shock front the electrostatic Langmuir waves (Z-mode) are generated and can be converted to the electromagnetic waves through direct linear mode conversion. 

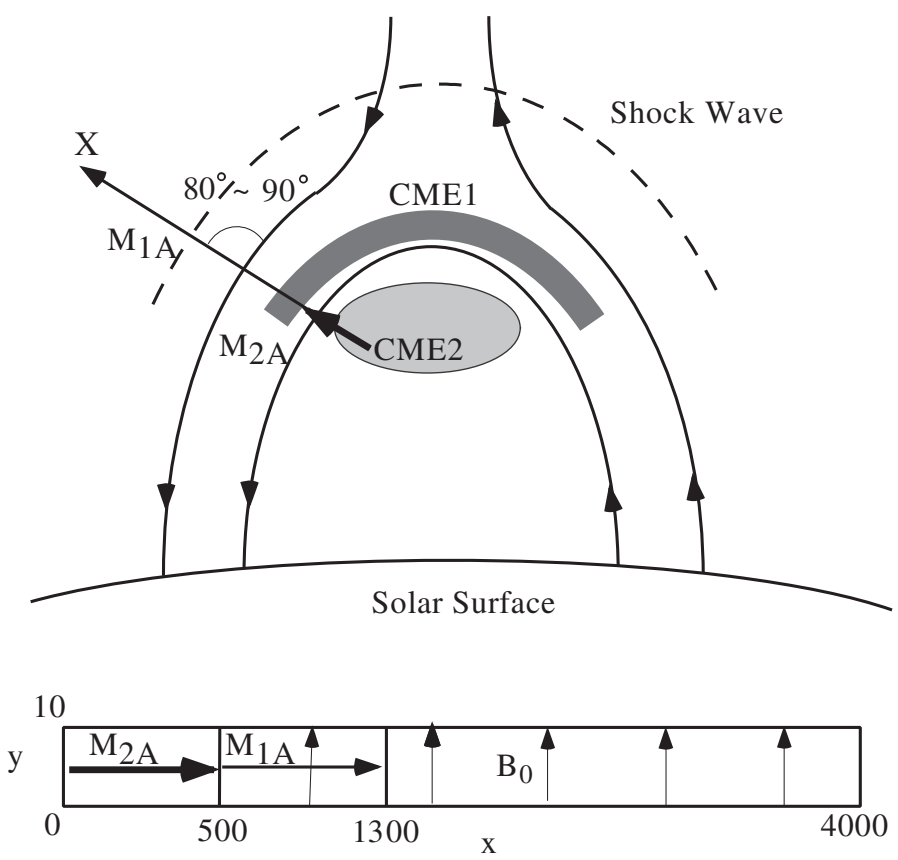

Fig. 1. The upper figure shows a schematic picture of the two coronal mass ejections associated with a shock wave: the fast CME (CME2) overtakes the slow CME (CME1) and they propagate at angles $\left(80^{\circ} \sim\right.$ $90^{\circ}$ ) to the local magnetic field. The lower figure shows the system size and coordinate system used in the simulation. The $x$-axis is the direction that two fast magnetosonic shocks propagate in the upper figure.

In the present paper we use a two-dimensional, fully relativistic PIC simulation to investigate how solar type II radio bursts can be enhanced when two fast magnetosonic shocks associated with CMEs collide. This work was motivated by recent observation by Gopalswamy et al. (2001) who reported the first detection at long radio wavelengths of interaction between CMEs in the interplanetary medium. They found that the radio signature is in the form of intense continuum-like radio emission following an interplanetary type II burst. At the time of radio enhancement, coronagraphic images showed a fast CME overtaking a slow CME. To simulate the above observation, we excited two fast magnetosonic shocks propagating perpendicularly and at an angle of $80^{\circ}$ to an ambient magnetic field. We looked at two cases: (1) one slow wave with the Alfvén Mach $M_{\mathrm{A}}=0.75$ and other fast shock with $M_{\mathrm{A}}=3.0$, and (2) one slow shock with the Alfvén Mach $M_{\mathrm{A}}=1.5$ and other fast shock with $M_{\mathrm{A}}=3.0$. The fast shock approaches the slow wave and overtakes it. We found that, during the collision of two shocks, the radio emission can be enhanced with the amplitude about ten times larger than the single type II burst, while the enhancement of the radio emission is about five times larger than the the single type II burst when a fast shock with Alfvén Mach $M_{\mathrm{A}}=3$ overtakes a slow shock with with Alfvén Mach $M_{\mathrm{A}}=1.5$. The simulation results agree well with the recent observations.

This paper is organised as follows. In Sect. 2 the simulation model and the parameters used in the simulations are presented. In Sect. 3 we show the simulation results, and in Sect. 4 we summarise our results.

\section{Simulation model}

In Fig. 1 we present a schematic picture of our simulation model. We assume that a fast CME is excited after a slow CME was first excited. The fast CME drives a fast magnetosonic shock wave with the Alfvén Mach $M_{\mathrm{A}}=3.0$, while the slow CME drives a fast magnetosonic shock wave with the Alfvén Mach $M_{\mathrm{A}}=1.5$, or a fast magnetosonic wave with the Alfvén Mach $M_{\mathrm{A}}=0.75$, a case that may correspond to the observations. Here we consider the case where the two shocks propagate perpendicularly and the other where they propagate with an angle of $80^{\circ}$ to an ambient magnetic field. The lower figure in Fig. 1 shows the system size and coordinate system used in the simulation. We used $2 \mathrm{D} 3 \mathrm{~V}$, the fully relativistic electromagnetic PIC code modified from the 3D3V TRISTAN code (Buneman 1993). The system size is $L_{x}=4000 \Delta$ and $L_{y}=10 \Delta$, where $\Delta(=1.0)$ is the grid size. We excite two fast magnetosonic shocks in the $x$-direction. The free boundary condition in the $x$ direction and the periodic boundary condition in the $y$ direction are imposed on particles and fields. We divided the simulation domain into three areas: left region $(x \leq 1000 \Delta)$, middle region (500 $\Delta<x \leq 1000 \Delta)$, and right region $(x>1000 \Delta)$. In the lefthand region, the average number of electron-ion pairs is 400 per cell, and the initial plasma flow velocity $v=0.24 c$ to drive a fast magnetosonic shock wave with Alfvén Mach, $M_{\mathrm{A}}=3$ is applied to the $x$-direction by the electric field $E_{z}$ through $E_{z}=v_{x} B_{y}$. This flow velocity is three times faster than Alfvén velocity $v_{\mathrm{A}}=0.078 c$. In the middle region, the average number of electron-ion pairs is 200 per cell, and the initial plasma flow velocity $v=0.12 c$ is also applied to the $x$-direction by the electric field $E_{z}$ through $E_{z}=v_{x} B_{y}$. This plasma flow velocity is 1.5 times faster than Alfvén velocity $v_{\mathrm{A}}=0.08 c$. And the external magnetic field $B_{y}=B_{0}$ is applied in the $y$-direction, that is, perpendicular to the flow direction. In the region on the right side, there are 100 electron-ion pairs per cell. The external magnetic field $B_{y}=B_{0}$ is also applied in the $y$-direction, that is, perpendicular to the flow direction.

The parameters used in this simulation are as follows. The ratio of ion mass to the electron mass is $m_{\mathrm{i}} / m_{\mathrm{e}}=64$, the ratio of electron cyclotron frequency to the plasma frequency is about $\omega_{\text {ce }} / \omega_{\text {pe }}=0.63$, plasma beta is $\beta=0.049$, skin depth is $c / \omega_{\text {pe }}=$ $10.0 \Delta$, electron thermal velocity is $v_{\text {th,e }}=0.1 c$, electron Larmor radius is $\rho_{\mathrm{e}}=1.58 \Delta$, ion Larmor radius is $\rho_{\mathrm{i}}=12.64 \Delta$, and the simulation time step is $\omega_{\mathrm{pe}} \Delta t=0.05$. The parameters $\omega_{\mathrm{ce}} / \omega_{\mathrm{pe}}$, $\beta, c / \omega_{\mathrm{pe}}, v_{\mathrm{th}, \mathrm{e}}, \rho_{\mathrm{e}}$, and $\rho_{\mathrm{i}}$ are calculated in the righthand region.

\section{Simulation results}

Here we present simulation results for the case of propagation angle with $80^{\circ}$, where the fast CME drives a fast magnetosonic shock wave with the Alfvén Mach $M_{\mathrm{A}}=3.0$, while the slow CME drives a fast magnetosonic wave with the Alfvén Mach $M_{\mathrm{A}}=0.75$. In Fig. 2 we present the time development of the magnetic field $B_{y}:$ (a) $\omega_{\mathrm{pe}} t=150$, (b) $\omega_{\mathrm{pe}} t=300$, (c) $\omega_{\mathrm{pe}} t=450$, (d) $\omega_{\mathrm{pe}} t=600$, (e) $\omega_{\mathrm{pe}} t=750$, and (f) $\omega_{\mathrm{pe}} t=900$. The fast shock with $M_{\mathrm{A}}=3.0$ (lefthand side) approaches the slow wave with $M_{\mathrm{A}}=0.75$ (righthand side). As seen in Figs. 1c and 1d, the fast shock wave overtakes the slow wave. After merging the shock strength is enhanced a little bit as seen in Figs. 1d-f.

Next we use the time development of the electric field $E_{z}$ to investigate how the electromagnetic wave emission from the shock front is enhanced. Figure 3 shows the time development of the electric field $E_{z}$ : (a) $\omega_{\mathrm{pe}} t=150$, (b) $\omega_{\mathrm{pe}} t=300$, (c) $\omega_{\mathrm{pe}} t=450$, (d) $\omega_{\mathrm{pe}} t=600$, (e) $\omega_{\mathrm{pe}} t=750$, and (f) $\omega_{\mathrm{pe}} t=900$. As seen in Fig. 3b, the wave emission is observed from the shock front. The emitted wave is an extraordinary electromagnetic wave (X-mode) (Sakai et al. 2005) that can be converted from the electrostatic Langmuir waves (Z-modes) by linear mode conversion through the density inhomogeneity. As seen in Figs. 3c, d 

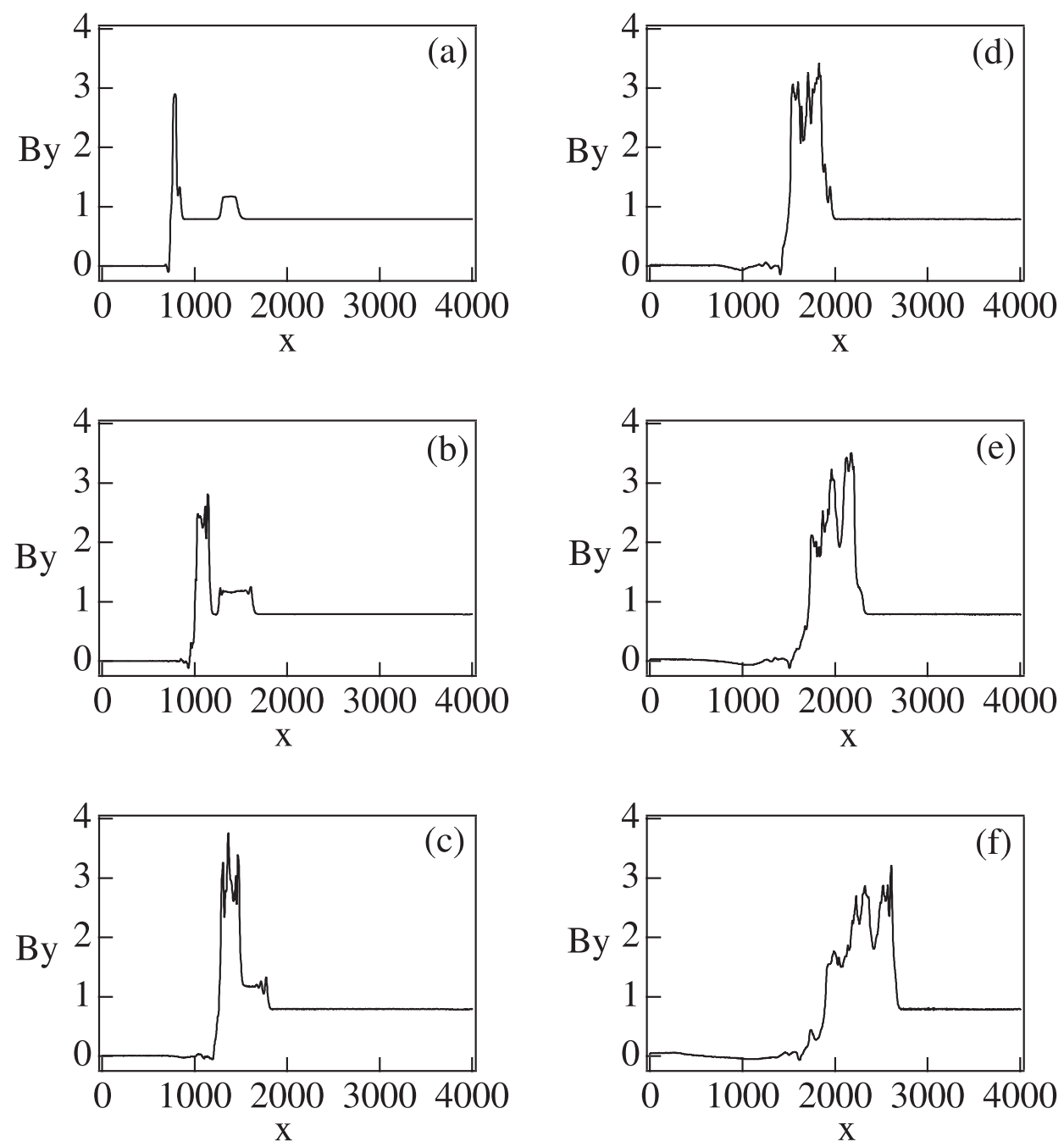

Fig. 2. Time development of magnetic field $B_{y}$ : a) $\omega_{\mathrm{pe}} t=150$, b) $\omega_{\mathrm{pe}} t=300$, c) $\omega_{\mathrm{pe}} t=450$, d) $\omega_{\mathrm{pe}} t=600$, e) $\omega_{\mathrm{pe}} t=750$, and f) $\omega_{\mathrm{pe}} t=900$.

showing that the fast shock overtakes the slow wave, the wave emission is strongly enhanced.

To understand the characteristics of the excited electromagnetic waves from the shocks, we performed two-dimensional Fourier transformation: one-space and one-time for $\omega_{\mathrm{pe}} t=200$ to $\omega_{\mathrm{pe}} t=404.8$ and $x=1900$ to $x=3024$ (before two shocks collide). Then, we obtained the dispersion relation of the $\mathrm{X}$-mode, as seen in Fig. 4a. We also attempted two-dimensional Fourier transformation: one-space and one-time for $\omega_{\mathrm{pe}} t=700$ to $\omega_{\mathrm{pe}} t=904.8$ and $x=2900$ to $x=3924$ (after two shocks collide). The obtained dispersion relation of the $\mathrm{X}$-mode is shown in Fig. 4b. Comparing two amplitudes of $E_{z}$ between before and after collision, which is measured by logarithmic scale, we found that the intensity of the X-mode after collision is about ten times higher than before collision. When comparing Figs. 4a and b, the excited X-mode has a broader frequency spectrum after the shock collision. This result is in good agreement with the observational result by Gopalswamy et al. (2001). Here we note that when a fast shock with Alfvén Mach $M_{\mathrm{A}}=3$ overtakes a slow shock with Alfvén Mach $M_{\mathrm{A}}=1.5$, the enhancement of the radio emission is about five times larger than the undisturbed type II burst.

We then investigate the mechanism to find out why exraordinary electromagnetic waves are strongly excited after the two shocks merge. Figure 5a shows the spatial distribution of electrostatic field $E_{x}$ at $\omega_{\mathrm{pe}} t=550$, when two shock waves begin to collide, and Fig. 5b shows the electron phase space of $v_{\mathrm{ex}} / c$ versus $x$ at $\omega_{\mathrm{pe}} t=550$. From these figures, we find that some electrons are accelerated associated with strong electric fields in the region between $x=1000$ and $x=2000$. These accerelated electrons may contribute to enhancing the electrostatic Langmuir waves (Z-modes). To understand the characteristics of the excited electrostatic waves from the merging region of two shocks, we performed two-dimensional Fourier transformation of $E_{x}$ : one-space and one-time for $\omega_{\mathrm{pe}} t=400$ to $\omega_{\mathrm{pe}} t=604.8$ and $x=1300$ to $x=1812$. Figure 6 shows the result of the dispersion relation for $E_{x}$.

The theoretical dispersion relation for obliquely propagating Langmuir wave ( $\mathrm{Z}$-mode) with Doppler-shifted frequency is given by

$\left(\omega-k v_{0}\right)^{2}=\omega_{\mathrm{pe}}^{2}+3 k^{2} v_{\mathrm{th}, \mathrm{e}}^{2}+\omega_{\mathrm{ce}}^{2} \sin ^{2} \theta$,

where $v_{\text {th,e }}$ is the electron thermal velocity and $\theta$ the angle between the wave-vector and the magnetic field. By using $v_{0}=$ $3 M_{\mathrm{A}}=3 \times 0.078 c, v_{\mathrm{th}, \mathrm{e}}=0.1 c$, and $\theta=80^{\circ}$, we plot the theoretical dispersion relation in Fig. 6, which agrees with the simulation result. Therefore we conclude that the electrostatic $\mathrm{Z}$-modes are excited in the merging region of two shocks. The excited-frequency region of the Z-modes coincides with the frequency region of the electromagnetic waves shown in Fig. 4b. 

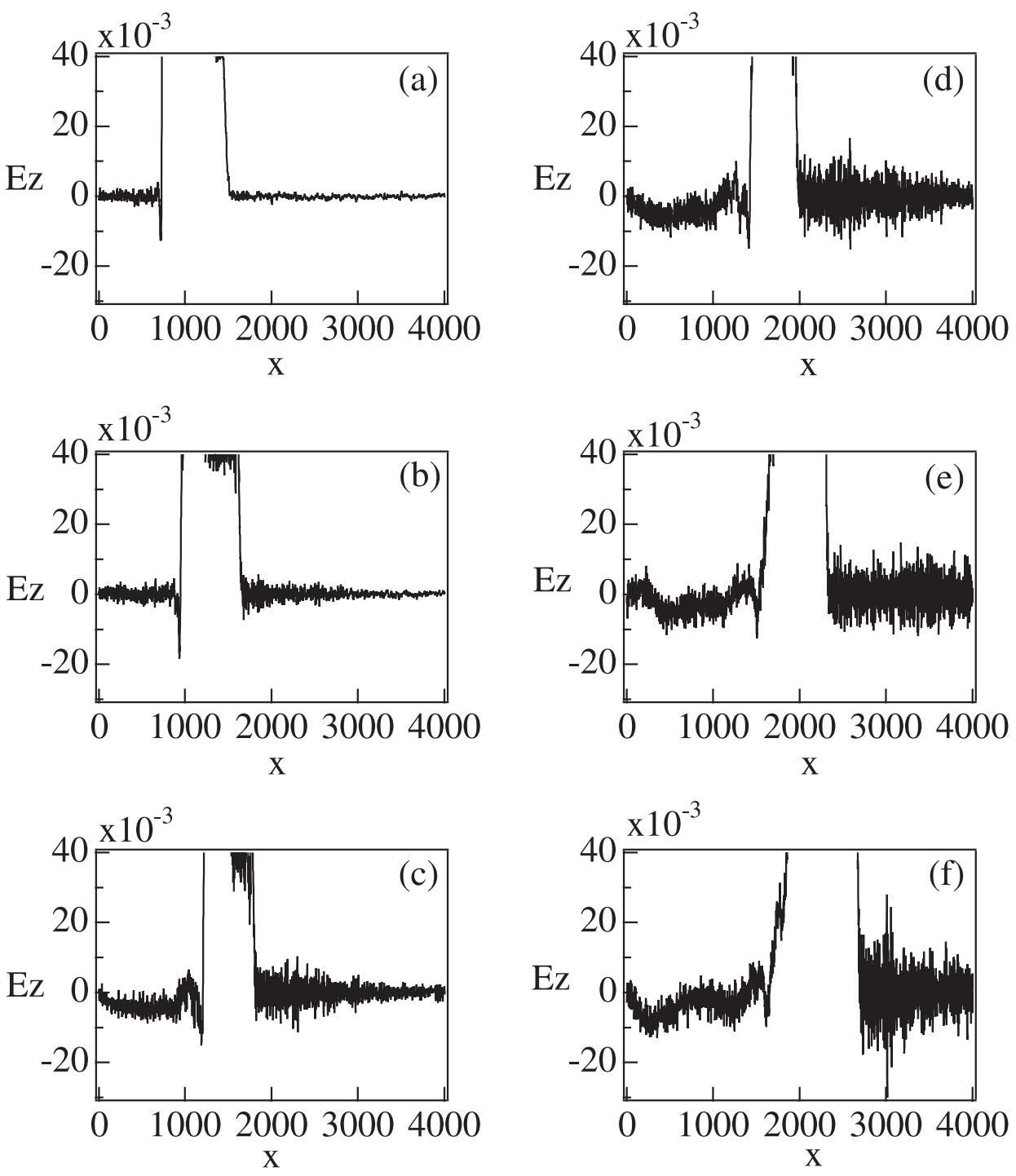

Fig. 3. Time development of electric field $E_{z}$ : a) $\omega_{\mathrm{pe}} t=150$, b) $\omega_{\mathrm{pe}} t=300$, c) $\omega_{\mathrm{pe}} t=450$, d) $\omega_{\mathrm{pe}} t=600$, e) $\omega_{\mathrm{pe}} t=750$, and f) $\omega_{\mathrm{pe}} t=900$.

In summary the excited Z-modes are generated by electron beams accelerated in the shock merging region and they could be converted to extra-ordinary electromagnetic waves through direct linear mode conversion.

Finally we show both electron and ion velocity distribution functions. Figure 7 shows the velocity distribution functions of electrons and Fig. 8 shows that of ions. Dotted lines in the figures denote initial velocity distributions (at $\left.\omega_{\mathrm{pe}} t=0\right)$ and solid lines denote the velocity distributions at the final step of simulation (at $\left.\omega_{\mathrm{pe}} t=1000\right)$. From these results, electrons are heated as a whole and slightly accelerated in the $+x$-direction. These accelerated electrons correspond to electron beams observed in the merging region of two shocks. The ions are accelerated mostly in the $z$-direction. They are accelerated by the surfatron mechanism (Sagdeev 1966) as discussed by Sakai et al. (2005).

\section{Summary}

We have investigated how radio waves are strongly excited when two shock waves collide due to the CMEs by using a twodimensional, electromagnetic, relativistic PIC code. We found that during the collision between a fast shock with Alfvén Mach $M_{\mathrm{A}}=3$ and a slow wave with Alfvén Mach $M_{\mathrm{A}}=0.75$, the

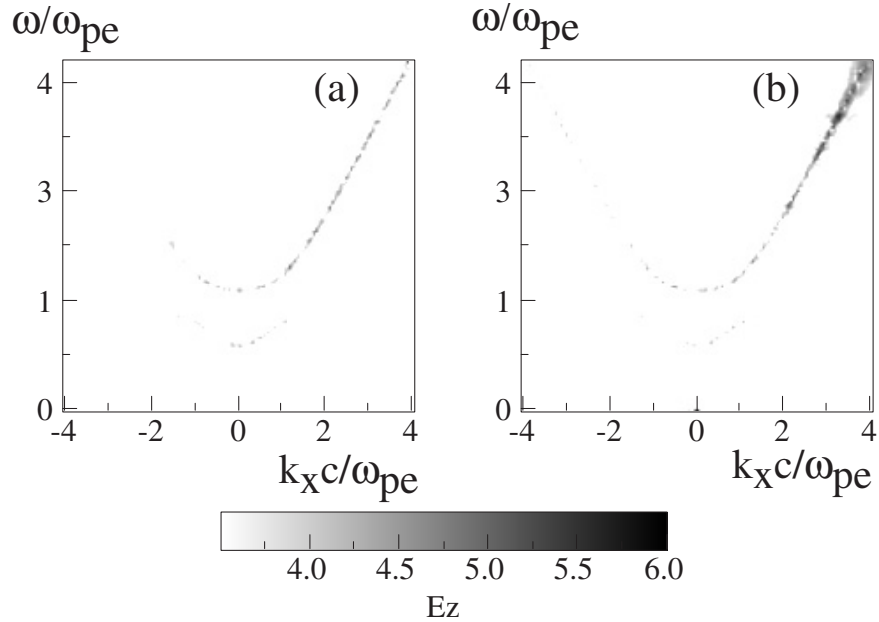

Fig. 4. The dispersion relations for $E_{z}$ obtained by space-time Fourier transformation: a) $1900 \leq x \leq 3024$ and $200 \leq \omega_{\text {pe }} t \leq 404.8$. b) $2900 \leq$ $x \leq 3924$ and $700 \leq \omega_{\mathrm{pe}} t \leq 904.8$.

radio emission with broad band can be enhanced with an amplitude about ten times larger than the undisturbed type II burst. 

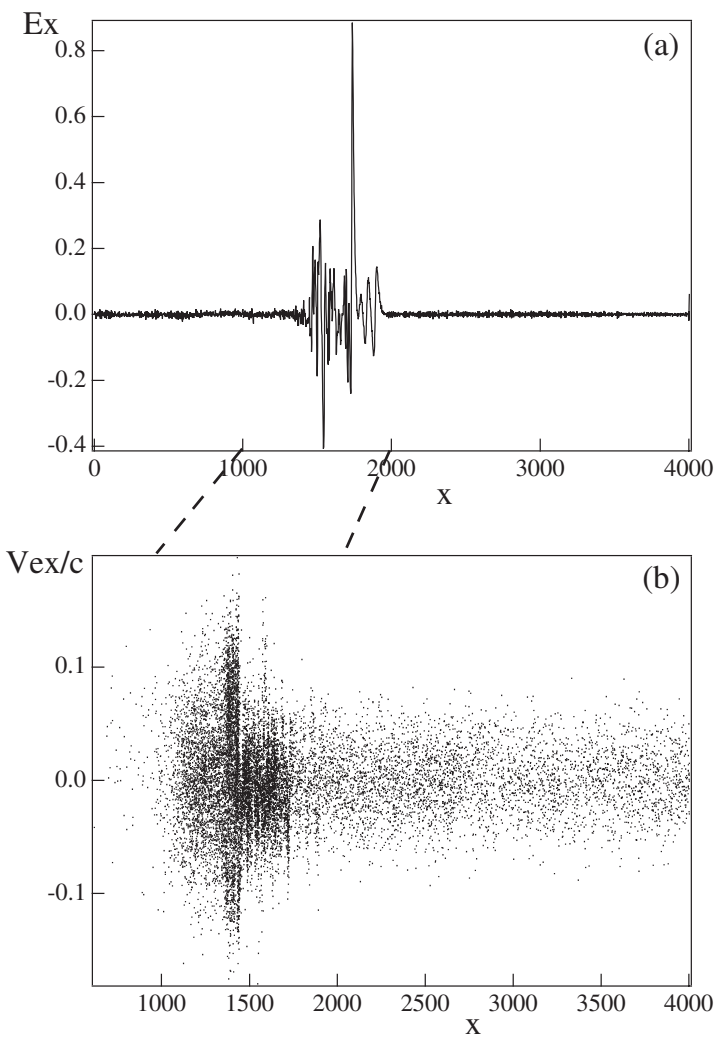

Fig. 5. Static components at $\omega_{\mathrm{pe}} t=550$ : a) spatial distribution of electric field $E_{x}$. b) The electron phase space plot $\left(v_{\mathrm{ex}}-x\right)$.

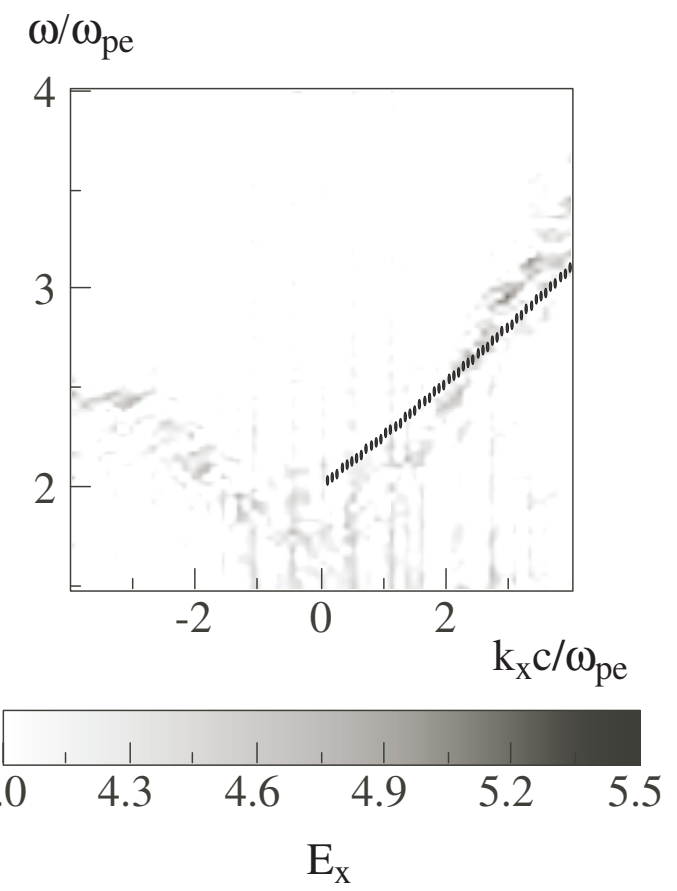

Fig. 6. The dispersion relation showing the excitation of the Z-mode. The dashed line shows the theoretical dispersion relation of the dopplershifted Z-mode.

We also found that when a fast shock with Alfvén Mach $M_{\mathrm{A}}=3$ overtakes a slow shock with Alfvén Mach $M_{\mathrm{A}}=1.5$, the enhancement of the radio emission is about five times larger than the the undisturbed type II burst. The amplification mechanism during the merging of the two shocks is caused by the enhancement of the electrostatic Langmuir waves (Z-modes) triggered
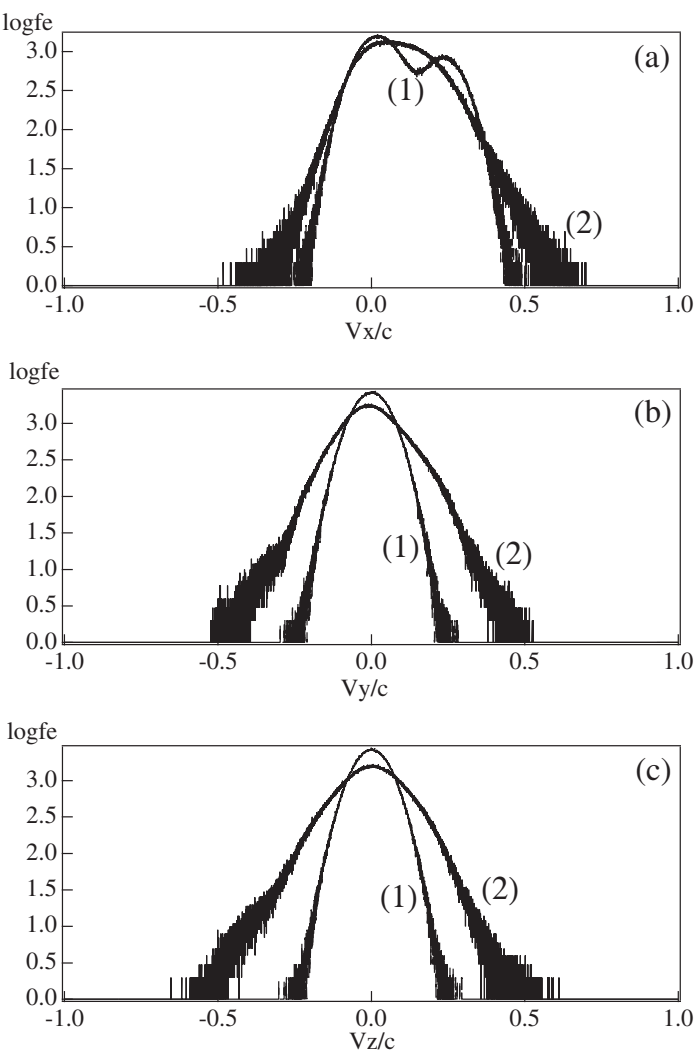

Fig. 7. The electron velocity distribution functions: a) $x$-component, b) $y$-component, and c) $z$-component. The dashed lines (1) are obtained by using data at $\omega_{\mathrm{pe}} t=0$, and the solid lines (2) are obtained by using data at $\omega_{\mathrm{pe}} t=1000$.
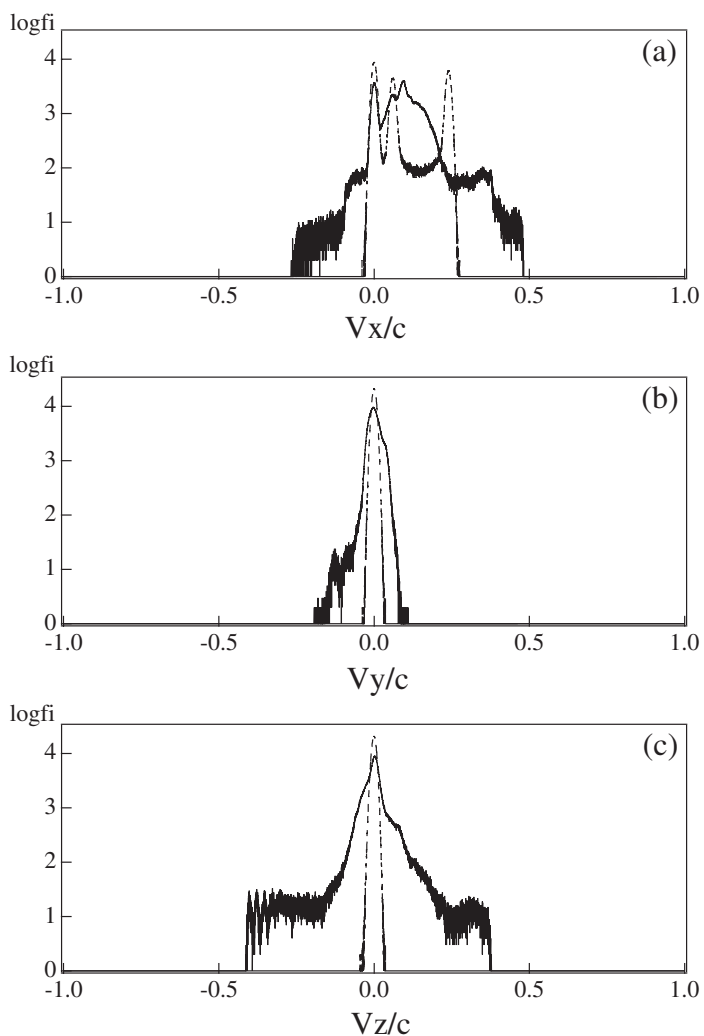

Fig. 8. The ion velocity distribution functions: a) $x$-component, b) $y$ component, and c) $z$-component. The dashed lines are obtained by using data at $\omega_{\mathrm{pe}} t=0$, and the solid lines are obtained by using data at $\omega_{\mathrm{pe}} t=$ 1000 . 
by the beam-like electrons accelerated in the shock-merging region. The obtained result agrees well with the recent observations by Gopalswamy et al. (2001).

Acknowledgements. We thank the anonymous referee for constructive comments.

\section{References}

Aschwanden, M. J. 2004, in Physics of The Solar Corona (Springer-Verlag), Chap. 15,657

Bale, S. D., Reiner, M. J., Bougeret, J.-L., et al. 1999, Geophy. Res. Lett., 26(11), 1573

Buneman, O. 1993, in Computer Space Plasma Physics, Simulation Techniques and Software, ed. H. Matsumoto, Y. Omura (Tokyo: Terra Scientific), 67

Cairns, I. H., Knock, S. A., Robinson P. A., \& Kuncic Z. 2003, Space Sci. Rev., 107,27
Goldman, M. V., \& Smith, D. F. 1986, in Physics of the SUN, ed. P. A. Sturrock, T. E. Holzer, D. M. Mihalas, \& R. K. Ulrich (D. Reidel Publ. Co.), 2 Chap. 15,325

Gopalswamy, N. Yashiro, S., Kaiser, M. L. Howard, R. A., \& Bougeret, J.-L. 2001, ApJ, 548, L91

Mann, G., \& Klassen, A. 2005, A\&A, 441, 319

Mann, G., Classen, T., \& Aurass, H. 1995, A\&A, 295, 775

Melrose, D. 1980, Aust. J. Phys., 33, 121

Sagdeev, R. Z. 1966, Reviews of Plasma Physics, ed. M. A. Leontovich (New York: Cosultants Bureau), 4, 23

Sakai, J. I., Mori, T., \& Saito, S. 2005, A\&A, 442, 687

Simnett, G. M. 2003, Sol. Phys., 213, 387

Simnett, G. M. 1, Sakai J. I., \& Forsyth, R. J. 2005, A\&A, 440, 766

Smith, D. F. 1970, Adv. Astro. Astrophys., 7, 147

Vršnak, B., Aurass, H., Magdalenić, J., \& Gopalswamy, N. 2001, A\&A, 377, 321

Vršnak, B., Magdalenić, J., Aurass, H., \& Mann, G. 2002, A\&A, 396, 673

Zlotnik, E.Ya., Klassen, A., Klein, K.-L., Aurass, H., \& Mann, G. 1998, A\&A, 331,1087 